\title{
Protective effects of metformin against myocardial ischemia-reperfusion injury via AMPK-dependent suppression of NOX4
}

\author{
YAN SHI and SHU-AI HOU \\ Department of Critical Care Medicine, People's Hospital of Rizhao, Rizhao, Shandong 276800, P.R. China
}

Received September 6, 2018; Accepted September 1, 2020

DOI: $10.3892 / \mathrm{mmr} .2021 .12351$

\begin{abstract}
Numerous studies have demonstrated that metformin can reduce the incidence of myocardial infarction and improve the prognosis of patients. However, its specific mechanism has not been determined. Using a rat model of myocardial ischemia-reperfusion injury (MIRI), it was observed that metformin significantly reduced infarct size, and decreased the levels of plasma lactate dehydrogenase and creatine kinase-MB form. A TTC-Evans blue staining was used to detect the infarct size and MTT assay was used to evaluate the cell viability. TUNEL assay was performed to evaluate apoptosis. Furthermore, 4-hydroxynonenal was detected by immunohistochemical staining. mRNA expression levels were detected by reverse transcription-quantitative PCR; protein expression levels were detected by immunoblotting. When treated with metformin, the number of TUNEL-positive cells was significantly decreased. Reduced $4 \mathrm{HNE}$ immunoreactivity was observed in metformin-treated rats as determined via immunohistochemistry. Furthermore, NADPH oxidase 4 (NOX4) was downregulated by metformin at both the mRNA and protein levels, and adenosine 5'-monophosphate-activated protein kinase (AMPK) phosphorylation was increased by metformin. In a primary myocardial hypoxia-reoxygenation cell model, metformin increased the viability of cardiomyocytes and reduced the content of malondialdehyde. It was also found that metformin upregulated the phosphorylation of AMPK and decreased the expression of NOX4. Furthermore, pre-treatment with AMPK inhibitor compound-C could block the effect of metformin, indicated by increased NOX4 compared with metformin treatment alone. These results suggested that metformin was capable of reducing the oxidative stress injury induced by MIRI. In conclusion, the present study indicated that metformin activated AMPK to inhibit
\end{abstract}

Correspondence to: Dr Yan Shi, Department of Critical Care Medicine, People's Hospital of Rizhao, 126 Tai-an Road, Donggang, Rizhao, Shandong 276800, P.R. China

E-mail: shiyanqd@126.com

Key words: metformin, adenosine 5'-monophosphate-activated protein kinase, NADPH oxidase 4, apoptosis, myocardial reperfusion injury, oxidative stress the expression of NOX4, leading to a decrease in myocardial oxidative damage and apoptosis, thus alleviating reperfusion injury.

\section{Introduction}

Coronary artery disease is severe, with high morbidity and mortality worldwide (1). Early reperfusion with thrombolysis or percutaneous coronary intervention is an effective strategy for acute myocardial infarction, and can effectively reduce infarct size and improve clinical outcome (2). However, further myocardial damage may occur during the reconstruction of coronary blood flow, called myocardial ischemia-reperfusion injury (MIRI). Mechanisms involved in MIRI include oxidative stress, myocardial apoptosis, calcium overload and mitochondrial dysfunction (3).

Oxidative stress refers to the accumulation of reactive oxygen species (ROS) and oxidative damage in cells (4). A previous study reported that NADPH oxidase (NOX)2 and NOX4 are major sources of cardiac ROS, playing an important regulatory role in the proliferation and death of cardiomyocytes (5). Inhibition of NOX2 and NOX4 contributes to decreased ROS and alleviation of MIRI (6). However, small quantities of ROS produced by NOX2 and NOX4 exert an anti-inflammatory protective effect during ischemia and reperfusion (5). In this scenario, inhibition of both NOX2 and NOX4 may aggravate reperfusion injury (7). A study on adenosine 5'-monophosphate-activated protein kinase (AMPK) knockout mice confirmed that the activation of AMPK in MIRI plays an important role in cardioprotection (8), but other studies have failed to get the same result $(9,10)$.

Metformin is commonly used for the treatment of type II diabetes (11). It can significantly reduce the risk of myocardial infarction and all-cause mortality in patients with type II diabetes (12). Therefore, it has been suggested that metformin, besides glycemic control, has an additional role in improving the prognosis of cardiovascular disease (13). In a rat model of myocardial infarction, metformin is reported to significantly reduce infarct size (14), while in a mouse model, a lower dose of metformin was administered $18 \mathrm{~h}$ prior to ischemia, which also reduced infarct size by 50\% (15); however, metformin did not affect plasma glucose concentration. In a subsequent isolated rat heart study, intracoronary infusion of metformin within the first $15 \mathrm{~min}$ of the reperfusion period reduced the 
infarct size by $40-50 \%$ (16). These results have been validated in vivo in rats and mice $(15,16)$. Various studies have reported that the cardioprotective effect of metformin is at least partly dependent on the activation of AMPK $(14,17)$. Although the effect of metformin on MIRI has been established, the mechanism involved has not been clarified. Concerning the antioxidant effects of metformin, studies have focused on whether it can reduce ROS synthesis, thereby reducing tissue fibrosis $(11,18)$. It is reported that metformin inhibits cardiac fibrosis by inhibiting NOX activity and reducing the production of ROS (19). For downstream signals, several studies have shown that AMPK inhibits NOX4 activity and reduces ROS production in a high-glucose-induced apoptosis model $(20,21)$. There is no clear explanation for the relationship between metformin and NOX4 in MIRI. Therefore, it was hypothesized that metformin inhibits NOX4 activity by activating AMPK, thereby reducing ROS production, ultimately playing a protective role against MIRI.

The present study aimed to elucidate whether metformin decreases MIRI by inhibiting NOX4 in rat MIRI and neonatal ventricle myocyte (NRVM) models, and to identify potential drug targets.

\section{Materials and methods}

Animals and materials. Sprague-Dawley (SD) male rats (weight, 200-250 g; age, 6-8 weeks; $\mathrm{n}=50$ ) and neonatal SD rats (age, 1-3 days; n=30) were obtained from the Animal Experimental Center of Shandong University. Protocols for the care and use of laboratory animals were approved by the Local Ethics Committee at the Medical College of Shandong University. Laboratory animals were reared according to the Guide for the Care and Use of Laboratory Animals from the National Institutes of Health (NIH) (22). At the end of animal experiments, rats were euthanized using excessive anesthesia administration as conditionally acceptable (intraperitoneal injection with sodium pentobarbital at a dosage of $200 \mathrm{mg} / \mathrm{kg}$ ). Metformin was purchased from Sigma-Aldrich (Merck KGaA).

Myocardial reperfusion injury model. Rats were anesthetized by sodium pentobarbital $(50 \mathrm{mg} / \mathrm{kg})$ via intraperitoneal injection and then ventilated with a rodent respirator (ALCV9A; Shanghai Alcott Biotech Co., Ltd.) after intubation. Then, the heart was exposed, and the left anterior descending coronary artery (LAD) was ligated by a 6-0 silk suture for $45 \mathrm{~min}$. Ischemia was determined by blanching of the myocardium, dyskinesia of the ischemic region and ST segment elevation on the ECG. Then, the heart was reperfused for $4 \mathrm{~h}$ by loosening the knot, and a marked hyperemic response indicated reperfusion (16).

Experimental protocol in vivo. The rats were randomly divided into three groups: i) Sham (sham operated) group, in which the heart was exposed but the LAD was not occluded; ii) ischemia-reperfusion (IR) group, in which the LAD was occluded for $45 \mathrm{~min}$ and reperfused for $4 \mathrm{~h}$; and iii) IR + metformin (IR + met) group, in which the LAD was occluded for $45 \mathrm{~min}$, and metformin $(5 \mathrm{mg} / \mathrm{kg})$ was intravenously injected via the jugular vein, subsequently followed by $4 \mathrm{~h}$ of reperfusion.
The dose of metformin used in the present study was selected based on a previous study (16).

Assessment of infarct size. Staining was performed as previously described (23). At the end of the reperfusion, the LAD was ligated again, and the heart was retrogradely infused with Evans blue $(0.25 \%$ in phosphate buffer; Sigma-Aldrich; Merck KGaA) from the aorta. The non-ischemic area was stained blue, indicating the area at risk (AR, non-blue region). Then, the heart was frozen at $-20^{\circ} \mathrm{C}$ for $30 \mathrm{~min}$ and sectioned into 6 slices $(2 \mathrm{~mm} / \mathrm{slice})$. The slices were incubated in triphenyltetrazolium chloride (TTC; $1 \%$ in phosphate buffer; Sigma-Aldrich; Merck KGaA) for $10 \mathrm{~min}$ at $37^{\circ} \mathrm{C}$ and then immersed in $4 \%$ paraformaldehyde for $4 \mathrm{~h}$ at room temperature. TTC staining can differentiate the infarct size (IS; white region) from the non-infarct area at risk (AR; red region). These heart slices were used only for Evans-blue-TTC staining. Finally, the slices were arranged from apex to base and digitally photographed. Digital images of the slices were analyzed using ImageJ software (version 1.47; National Institutes of Health). The final result was presented as the IS/AR as previously described (24).

Evaluation of apoptosis. The left ventricles of rat hearts were fixed in $4 \%$ paraformaldehyde at room temperature for $24 \mathrm{~h}$ and embedded in paraffin at $56^{\circ} \mathrm{C}$ for $1.5 \mathrm{~h}$, then sectioned (thickness, $6 \mu \mathrm{m}$ ). According to the manufacturer's instructions, terminal deoxynucleotidyl transferase-mediated dUTP nick end labelling (TUNEL) assays were performed to detect apoptotic cells in heart tissue sections using an in situ cell death detection kit (Roche Applied Science). The sections were incubated in TUNEL reaction mixture for $60 \mathrm{~min}$ at $37^{\circ} \mathrm{C}$ in a humidified atmosphere in the dark. Sections were then stained with hematoxylin (Beijing Solarbio Science \& Technology, Co., Ltd.) for $2 \mathrm{~min}$ at room temperature, washed with PBS, and mounted with mounting medium (Abcam). The nuclei were counted in 10 random fields of each section using a light microscope (magnification, x400; Leica Microsystems GmbH), and the results are shown as a percentage of TUNEL-positive nuclei compared with the total number of cell nuclei.

Western blotting. RIPA solution (Abcam) was used to homogenize the frozen tissue samples and cultured cardiomyocytes and BCA assay (Pierce; Thermo Fisher Scientific, Inc.) was used to detect the concentration of protein samples. Then, $50 \mu \mathrm{g} /$ lane extracted protein was separated via $10 \%$ SDS-PAGE and transferred onto PVDF membranes (EMD Millipore). Non-specific reactivity was blocked with $5 \%$ milk for $2 \mathrm{~h}$ at room temperature and the membrane was incubated with primary antibody overnight at $4{ }^{\circ} \mathrm{C}$ in buffer $(10 \mathrm{mM}$ Tris-HCl; pH 7.5; 150 mM NaCl, 2\% Tween-20, 4\% bovine serum albumin). The membrane was then incubated with secondary antibody for $1.5 \mathrm{~h}$ at room temperature. ECL chemiluminescence (Cell Signaling Technology, Inc.) was used for detection by an imaging system (ChemiDoc XRS; Bio-Rad Laboratories, Inc.), and densitometry was performed using ImageJ software (version 1.47; National Institutes of Health) semi-quantitatively (4). All protein levels were normalized to that of GAPDH. The antibodies used in the study were as follows: Phosphorylated (p)-AMPK (Thr172; 
1:1,000; cat. no. 50081; rabbit monoclonal antibodies; Cell Signaling Technology, Inc.), AMPK (1:1,000; cat. no. 2532; rabbit monoclonal antibodies; Cell Signaling Technology, Inc.) and NOX4 (1:1,000; cat. no. ab109225; rabbit monoclonal antibody; Abcam); GAPDH (1:10,000; cat. no. AB2302; mouse monoclonal antibody; EMD Millipore) and corresponding horseradish peroxidase (HRP)-conjugated secondary antibodies (OriGene Technologies, Inc.).

Isolation of neonatal rat cardiomyocytes. NRVMs were isolated from 1-3-day-old SD rats as previously described (25). The rats were euthanized by decapitation and the hearts were rapidly excised, minced and dissociated with $0.1 \%$ trypsin and $0.03 \%$ collagen II solution. The dispersed cells were then plated at a density of $2 \times 10^{5}$ cells $/ \mathrm{cm}^{2}$ on a 6 -well plate with $2 \mathrm{ml} /$ well DMEM supplemented with $10 \%$ fetal bovine serum (FBS; both HyClone; GE Healthcare Life Sciences). Cytosine arabinoside $(10 \mathrm{M})$ was used to suppress non-cardiomyocytes. After serum-starved cultivation $\left(37^{\circ} \mathrm{C}, 95 \% \mathrm{O}_{2}, 5 \% \mathrm{CO}_{2}, 24 \mathrm{~h}\right)$, the myocytes were put in a hypoxia $\left(95 \% \mathrm{~N}_{2}, 5 \% \mathrm{CO}_{2}\right)$ incubator for $4 \mathrm{~h}$ at $37^{\circ} \mathrm{C}$ then, the culture medium was replaced with fresh oxygenated DMEM (10\% FBS) and the plates were transferred to a normoxic incubator $\left(5 \% \mathrm{CO}_{2}\right)$ for $6 \mathrm{~h}$ of reoxygenation.

Experimental protocol in vitro (i). The NRVMs were randomly divided into four groups: i) Control (CON) group, cells were incubated with normal oxygen; ii) hypoxia-reoxygenation (HR) group, in which the cells were incubated in hypoxic conditions for $4 \mathrm{~h}$ and reoxygenated for $6 \mathrm{~h}$; iii) HR + metformin $(\mathrm{HR}+\mathrm{met})$ group, at the onset of reoxygenation, metformin $(0.1 \mathrm{mM})$ was added to the DMEM (the dose of metformin used in the present study was selected based on a previous study) (26); and iv) HR + metformin + compound-C (HR + met + compound-C), at the onset of reoxygenation, metformin and compound-C (10 nM; cat. no. ab120843; Abcam) were added into the medium simultaneously (the dose of compound-C used in the present study was selected based on a previous study) (26).

MTT assay. Cell viability was assessed by an MTT assay. The MTT assay was performed according to the manufacturer's protocols (cat. no. C0009; Beyotime Institute of Biotechnology). Cardiomyocytes were plated on 96-well dishes at a density of $1 \times 10^{4}$ cells/well. Following reoxygenation, cells were incubated with MTT reaction solution $(0.5 \mathrm{mg} / \mathrm{ml})$ for $4 \mathrm{~h}$ and the medium was removed. Then, $100 \mu \mathrm{l}$ DMSO was added to each well for $10 \mathrm{~min}$ and mixed thoroughly with a mechanical plate mixer. At last, the absorbance was measured with a microplate reader (Molecular Devices, LLC) at a wavelength of $530 \mathrm{~nm}$.

Plasma creatine kinase- $M B$ form $(C K-M B)$ and lactate dehydrogenase ( $\mathrm{LDH}$ ) activity. Blood samples were centrifuged at $1,800 \mathrm{x}$ g for $10 \mathrm{~min}$ at $4^{\circ} \mathrm{C}$. LDH (cat. no. A020-1) and CK-MB (cat. no. E006-1-1) levels in plasma were detected according to the manufacturer's protocols (Nanjing Jiancheng Bioengineering Institute). The optical density of the tetrazolium product was determined spectrophotometrically (Molecular Devices, LLC) at a wavelength of $490 \mathrm{~nm}$.
Malondialdehyde (MDA) detection. Lipid peroxidation was estimated by measuring the concentration of MDA in the isolated myocytes using a Lipid Peroxidation Assay kit (cat. no.MAK085; Calbiochem; Merck KGaA). Briefly, the cells were lysed and mixed with reagent R1 (N-methyl-2-phenylindole in acetonitrile and methanol) for a total of $60 \mathrm{~min}$ at $45^{\circ} \mathrm{C}$. The samples were then centrifuged at $21,130 \mathrm{x} \mathrm{g}$ for $10 \mathrm{~min}$ at room temperature. The optical density of the supernatant was determined spectrophotometrically at a wavelength of $586 \mathrm{~nm}$ (Molecular Devices, LLC). The MDA concentration was displayed as $\mu \mathrm{mol} / \mathrm{g}$ protein.

Immunohistochemistry. Tissue sections (thickness, $6 \mu \mathrm{m}$ ) were deparaffinized and then blocked with CAS-Block (Invitrogen; Thermo Fisher Scientific, Inc.) for $1 \mathrm{~h}$ at $37^{\circ} \mathrm{C}$. The sections were incubated with anti-4HNE primary antibody (1:200; cat. no. ab48506; Abcam) for $2 \mathrm{~h}$ at $37^{\circ} \mathrm{C}$ followed by incubation with HRP-conjugated secondary antibody (1:500; cat. no. 7074; Cell Signaling Technology, Inc.) for $1 \mathrm{~h}$ at $37^{\circ} \mathrm{C}$. Sections were developed in 3,3'-diaminobenzidine solution for $3 \mathrm{~min}$ at room temperature, then stained with hematoxylin for $2 \mathrm{~min}$ at room temperature, washed with PBS, and mounted with mounting medium. Sections were visualized under a light microscope (magnification, x400; Leica Microsystems GmbH).

$R N A$ isolation and reverse transcription-quantitative (RT-q) $P C R$. Total RNA of myocardial tissues and NRVMs was extracted using TRIzol ${ }^{\circledR}$ reagent (Invitrogen; Thermo Fisher Scientific, Inc.). The TaqMan ${ }^{\circledR}$ Reverse Transcription Reagents (cat. no. N8080234; Applied Biosystems; Thermo Fisher Scientific, Inc.) was used to generate the first-strand cDNA using the following thermocycling conditions: $70^{\circ} \mathrm{C}$ for $5 \mathrm{~min}, 42^{\circ} \mathrm{C}$ for $1 \mathrm{~h}$ and $70^{\circ} \mathrm{C}$ for $15 \mathrm{~min}$. A Mx3000 Multi-plex Quantitative PCR System (Stratagene; Agilent Technologies, Inc.) with SYBR-Green fluorescence (Molecular Probes, LLC) was used to amplify the cDNA in 35 cycles. Each cycle consisted of heating denaturation for $30 \mathrm{sec}$ at $94^{\circ} \mathrm{C}$, annealing for $30 \mathrm{sec}$ at $56^{\circ} \mathrm{C}$ and extension for $30 \mathrm{sec}$ at $72^{\circ} \mathrm{C}$. All samples were quantitated using the comparative $\mathrm{Cq}$ method for relative quantitation of gene expression, normalized to GAPDH (27). The primers used in this study were as follows: NOX4, forward, 5'-TGGCCAACGAAGGGGTT AAA-3' and reverse, 5'-CACTGAGAAGTTCAGGGCGT-3'; AMPK, forward, 5'-GATCGGACACTACGTGCTGG-3' and reverse, 5'-TAGTTGCTCGCTTCAAGGGG-3'; and GAPDH, forward, 5'-TGATGACATCAAGAAGGTGGTGAAG-3' and reverse, 5'- TCCTTGGAGGCCATGTAGGCCAT-3'.

Small interfering (si)RNA transfection. Transfection of siRNA was performed with Lipofectamine ${ }^{\circledR} 2000$ (Invitrogen; Thermo Fisher Scientific, Inc.) according to the manufacturer's protocols. NRVM $\left(6 \times 10^{5}\right.$ cells per well of a 6 -well plate at) were transfected with siRNA directed to AMPK (cat. no. sc-74461; Santa Cruz Biotechnology, Inc.) at $10 \mu \mathrm{M}$ for $6 \mathrm{~h}$ in serum-free medium. Afterwards, it was changed to standard medium containing $10 \%$ FBS for $24 \mathrm{~h}$. Non-specific (NS)-siRNA (cat. no. sc-37007; Santa Cruz Biotechnology, Inc.) was used as negative control.

Experimental protocol in vitro (ii). The NRVMs were randomly divided into four groups: i) $\mathrm{CON}+\mathrm{NS}$-siRNA group, in which 
A

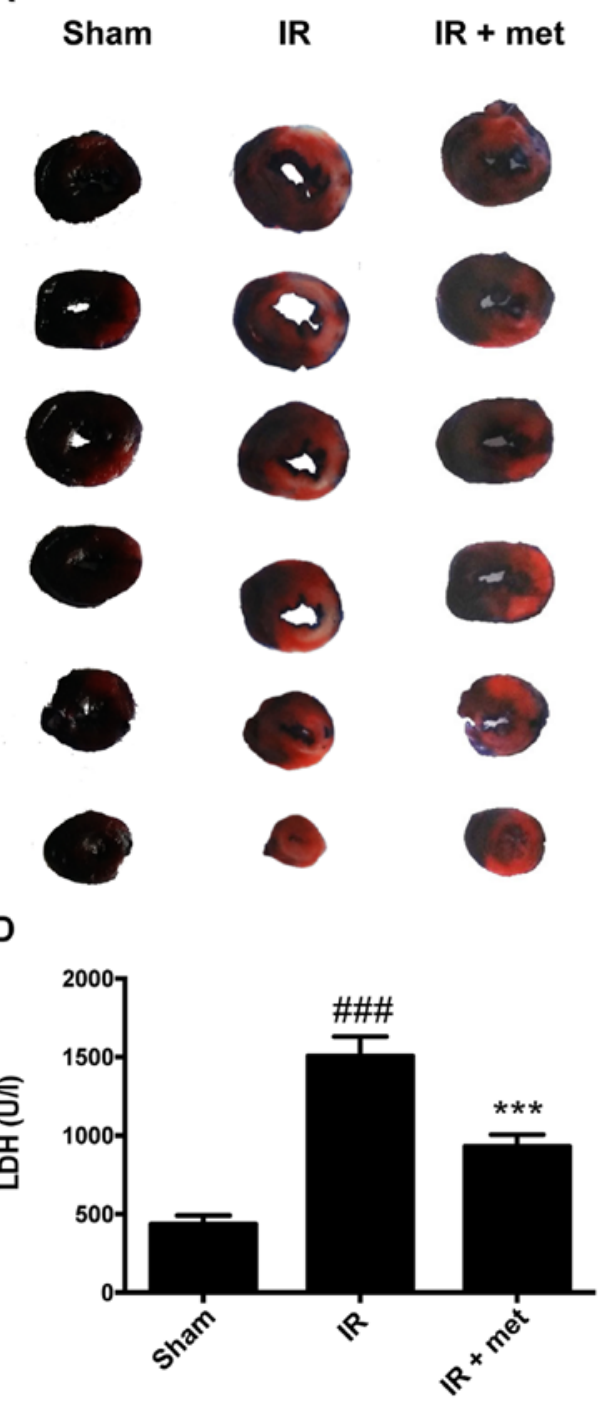

B

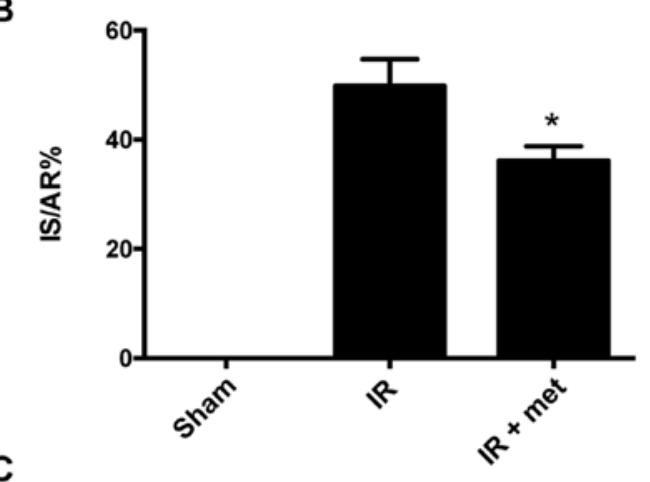

C
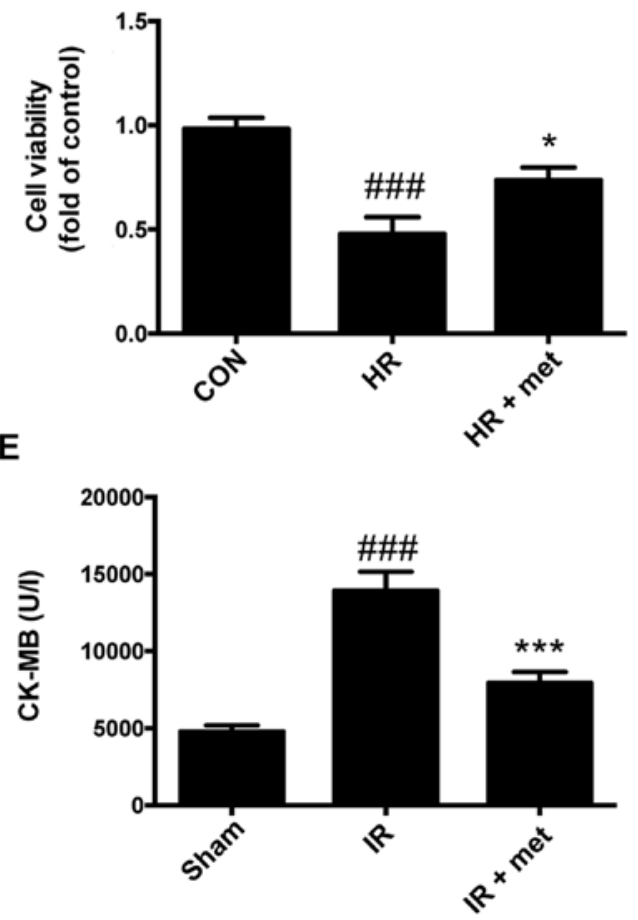

Figure 1. Cardioprotective effects of metformin against reperfusion injury. (A) Representative heart slices stained by Evans blue and triphenyltetrazolium chloride. Blue, non-blue and white areas represent non-ischemic, AR and IS areas. (B) IS/AR scores in different groups. (C) Cell viability detected by an MTT assay. ${ }^{\# \# \#} \mathrm{P}<0.001$ vs. $\mathrm{CON} ;{ }^{*} \mathrm{P}<0.05$ vs. HR. (D) Plasma LDH concentration. (E) Plasma CK-MB concentration. Data are shown as the mean \pm SEM (n=6).

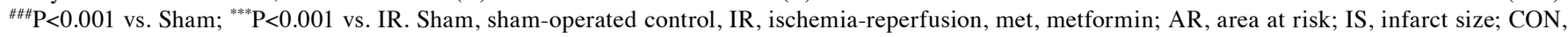
control; HR, hypoxia-reoxygenation; LDH, lactate dehydrogenase; CK-MB, creatine kinase MB form.

the cells were pretreated with NS-siRNA and then incubated with normal oxygen; ii) HR + NS-siRNA group, in which the cells were pretreated with NS-siRNA and then subjected to $4 \mathrm{~h}$ of hypoxia and $6 \mathrm{~h}$ of reoxygenation; iii) $\mathrm{HR}+$ metformin + NS-siRNA $(\mathrm{HR}+$ met + NS-siRNA) group, in which the cells were pretreated with NS-siRNA and then subjected to $4 \mathrm{~h}$ of hypoxia and $6 \mathrm{~h}$ of reoxygenation, and at the onset of reoxygenation, metformin $(0.1 \mathrm{mM})$ was added; and iv) $\mathrm{HR}$ + metformin + AMPK-siRNA (HR + met + AMPK-siRNA) group, in which the cells were pretreated with AMPK-siRNA and then subjected to $4 \mathrm{~h}$ of hypoxia and $6 \mathrm{~h}$ of reoxygenation, and at the onset of reoxygenation, metformin $(0.1 \mathrm{mM})$ was added into DMEM.

Statistical analysis. All data are presented as the mean \pm SEM $(n \geq 3)$. Statistical comparisons between the groups were performed using one-way ANOVA followed by Newman-Keuls or Tukey's post hoc test. Kruskal-Wallis test followed by
Dunn's post hoc test was used for non-parametric data. The statistical analyses were performed by GraphPad Prism 5.0 (GraphPad Software, Inc.). P $\leq 0.05$ was considered to indicate a statistically significant difference.

\section{Results}

Cardioprotective effects of metformin. After $24 \mathrm{~h}$ reperfusion in IR rat, the IS and AR were determined using Evans blue and TTC. The IS/AR was used to evaluate damage to the heart as previously described (16). The results showed that treatment with metformin significantly reduced the infarct size in IR rats by $\sim 20 \%(\mathrm{P}<0.05$; Fig. $1 \mathrm{~A}$ and $\mathrm{B})$. There was no infarct area in the Sham group, so the IS/AR was 0. Plasma LDH and CK-MB levels in the IR group were increased compared with the Sham group; however, the increases were significantly attenuated by metformin $(\mathrm{P}<0.001$; Fig. $1 \mathrm{D}$ and $\mathrm{E})$. The viability of cardiomyocytes decreased significantly after HR treatment 
A
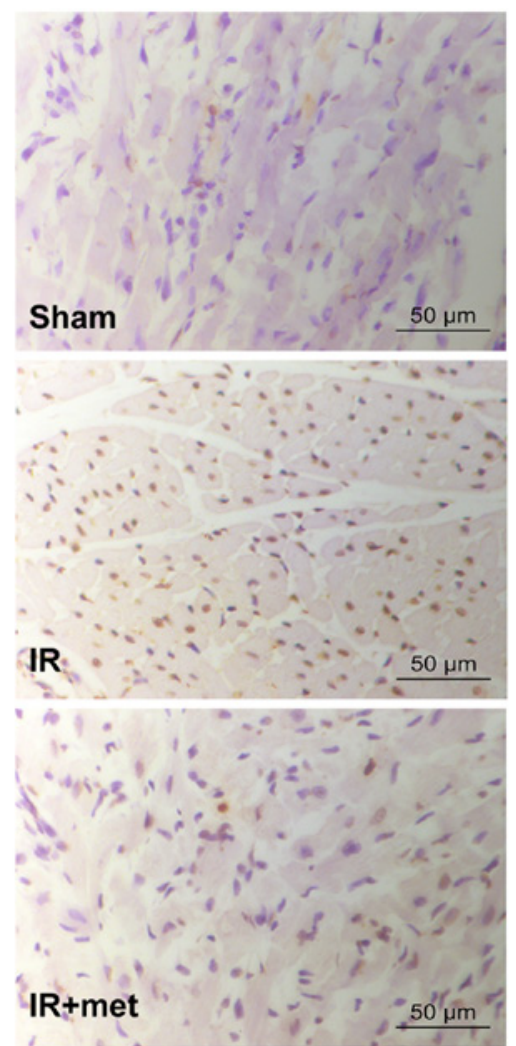

C

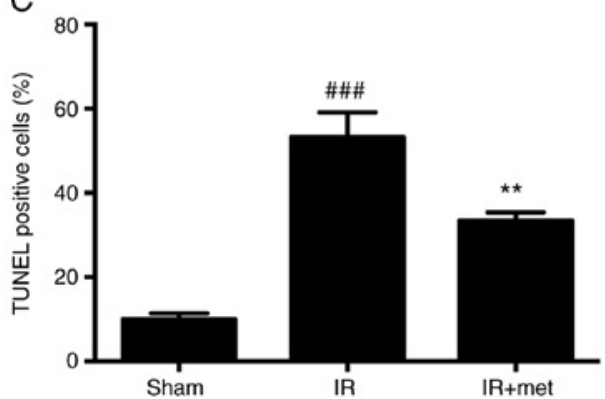

B
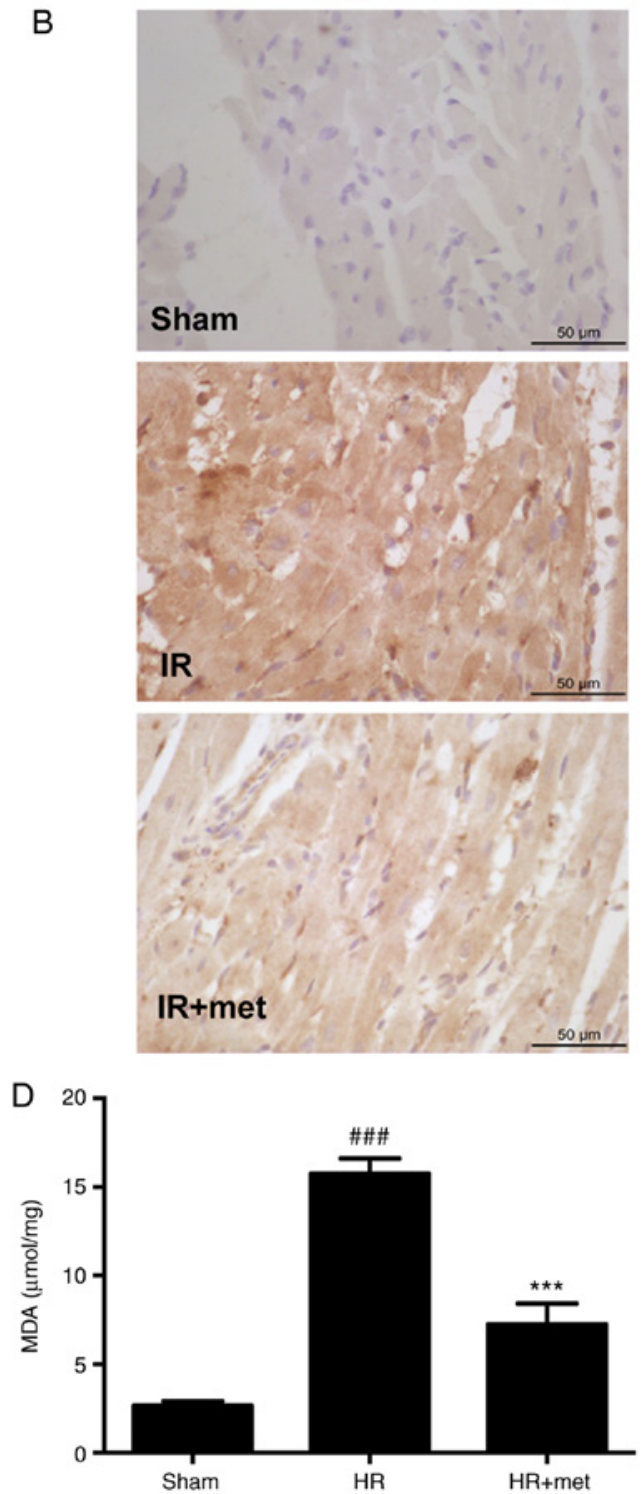

Figure 2. Alleviation of oxidative damage and apoptosis by metformin. (A) Representative myocardial apoptosis in paraffin sections of the heart in the risk area, as determined via a TUNEL assay; brown cells are TUNEL-positive. (B) Representative paraffin sections of the heart in the risk area stained for 4-hydroxynonenal. (C) Percentage of TUNEL-positive cells $(n=6) .{ }^{\# \# \#} \mathrm{P}<0.001$ vs. Sham; ${ }^{* *} \mathrm{P}<0.01$ vs. IR. (D) Concentration of MDA in cardiomyocytes ( $=6$ ). Data are shown as the mean \pm SEM. ${ }^{\# \#} \mathrm{P}<0.001$ vs. CON; ${ }^{* * *} \mathrm{P}<0.001$ vs. HR. Sham, sham-operated control; IR, ischemia-reperfusion; HR, hypoxia-reoxygenation; met, metformin; MDA, malondialdehyde; TUNEL, terminal deoxynucleotidyl transferase-mediated dUTP nick end labelling.

compared with the cells in CON group $(\mathrm{P}<0.001)$; treatment with metformin increased the viability of HR-treated cardiomyocytes $(\mathrm{P}<0.05$; Fig. 1C).

Metformin alleviates oxidative damage and apoptosis. Oxidative stress plays an important role in the process of MIRI (14). 4-hydroxynonenal (4HNE) is the final product of lipid oxidation, the amount of which indicates the severity of oxidative damage (28). Immunohistochemistry results showed that the levels of $4 \mathrm{HNE}$ in the IR group were notably increased compared with the Sham group (Fig. 2B). However, the content of $4 \mathrm{HNE}$ in the IR + met group was markedly decreased compared with the IR group (Fig. 2B). In addition to $4 \mathrm{HNE}$, another lipid peroxidation product, MDA, was measured in cells following HR. The results showed that the level of MDA in the HR + met group was significantly decreased compared with the HR group (Fig. 2D; P<0.001). A TUNEL assay was performed in tissue sections to evaluate apoptosis. It was found that apoptosis was increased in the IR group compared with the Sham group $(\mathrm{P}<0.05$; Fig. $2 \mathrm{~A}$ and $\mathrm{C})$. When treated with metformin, the percentage of TUNEL-positive cells was significantly decreased compared with the IR group $(\mathrm{P}<0.01$; Fig. 2C).

Metformin inhibits the expression of NOX4 and increases the activation of AMPK. NOX4 plays an important role in regulating the content of ROS in cardiac myocytes (6). A significant increase in NOX4 mRNA was observed in the IR group compared with the Sham group; after metformin was administered, the elevation in NOX4 was significantly attenuated $(\mathrm{P}<0.05$; Fig. $3 \mathrm{~A})$. The protein level of NOX4 in the IR group was also significantly increased compared with that in the Sham group; after metformin treatment, the increase of NOX4 was significantly reversed $(\mathrm{P}<0.05$; Fig. $3 \mathrm{~B}$ and $\mathrm{C})$. 
A

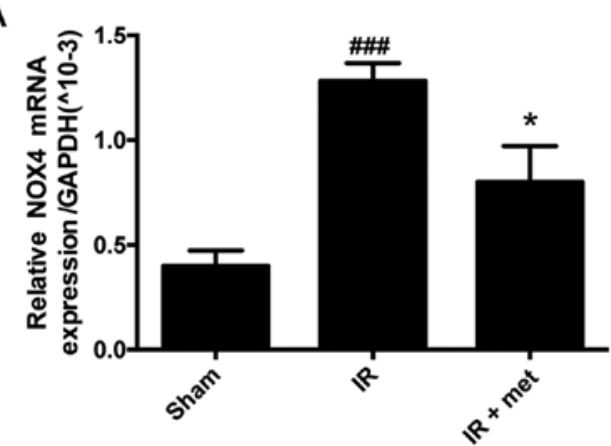

B

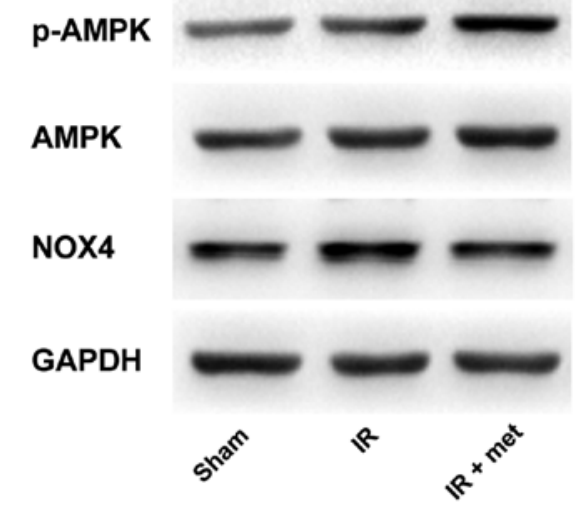

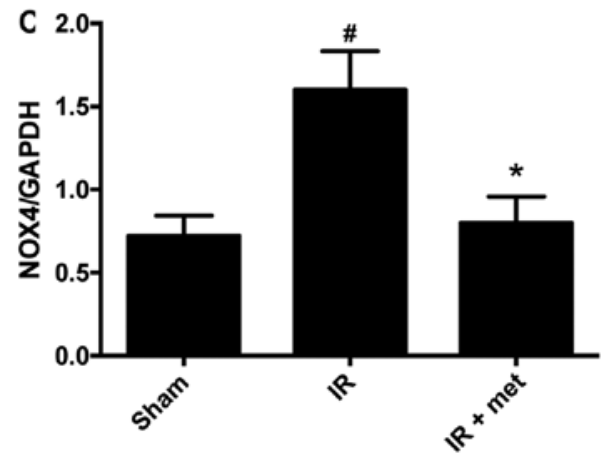

D

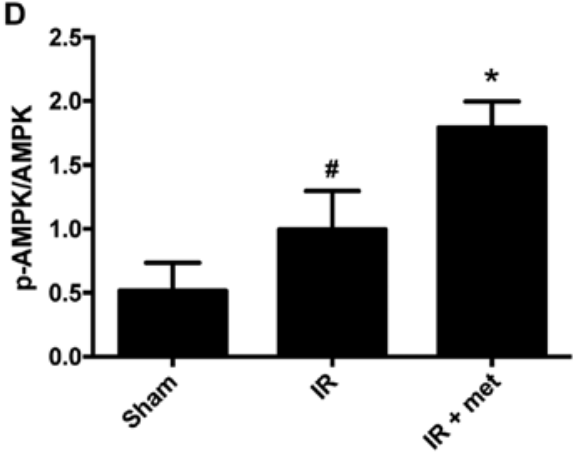

Figure 3. Effect of metformin on the activity of NOX4 and the activation of AMPK. (A) Expression of NOX4 mRNA as determined via reverse transcription-quantitative PCR. (B) Representative protein levels of p-AMPK, AMPK and NOX4 as determined via western blotting. (C) Semi-quantification of NOX4 protein levels. (D) Semi-quantification of the p-AMPK/AMPK ratio. Protein levels were normalized to GAPDH. Data are shown as the mean \pm SEM (n=6). ${ }^{\#} \mathrm{P}<0.05,{ }^{\# \# \#} \mathrm{P}<0.001$ vs. Sham; ${ }^{\mathrm{P}}<0.05$ vs. IR. Sham, sham-operated control; IR, ischemia-reperfusion; met, metformin; NOX, NADPH oxidase; AMPK, adenosine 5'-monophosphate-activated protein kinase; p, phosphorylated.
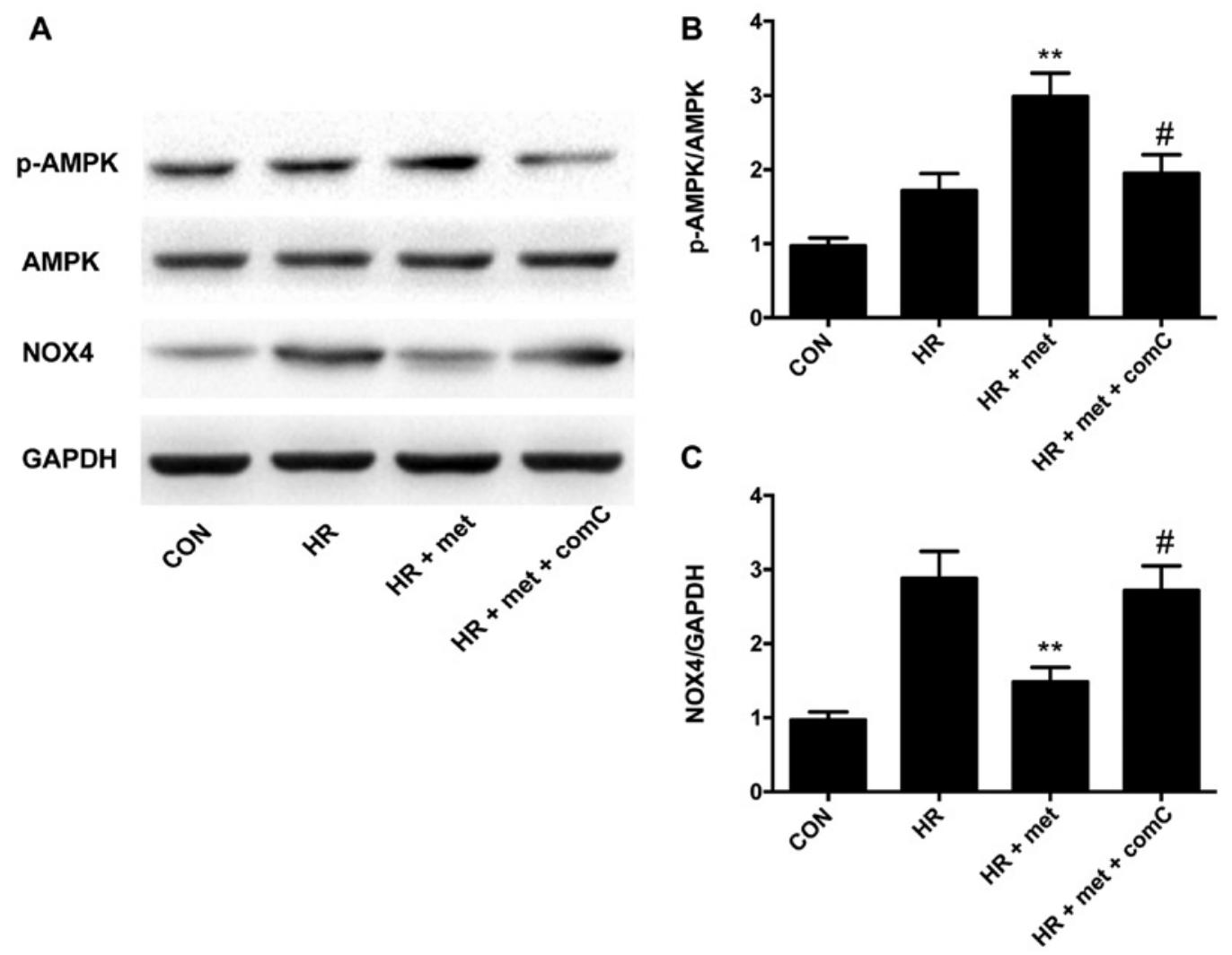

Figure 4. AMPK inhibitor upregulates the expression of NOX4 protein in vitro. (A) Representative protein levels of p-AMPK, AMPK and NOX4 as determined via western blotting. (B) Semi-quantification of the p-AMPK/AMPK ratio. (C) Semi-quantification of NOX4 protein levels. Protein levels were normalized to GAPDH. Data are shown as the mean \pm SEM $(n=6)$. ${ }^{* *} \mathrm{P}<0.01$ vs. HR; " $\mathrm{P}<0.05$ vs. HR + met. CON, control; HR, hypoxia-reoxygenation; met, metformin; comC, compound-C; NOX, NADPH oxidase; AMPK, adenosine 5'-monophosphate-activated protein kinase; p, phosphorylated. 

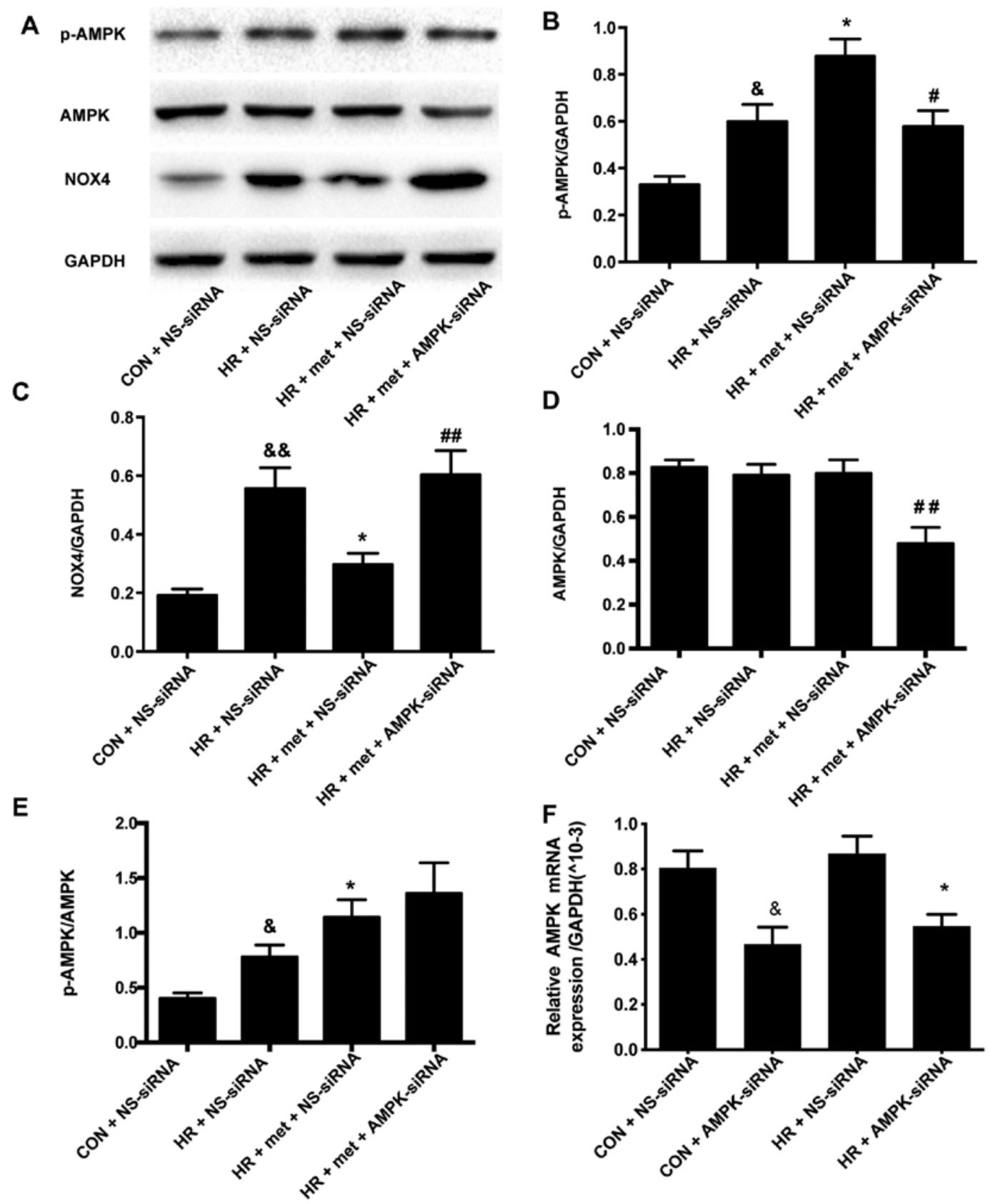

Figure 5. AMPK siRNA increases the expression of NOX4 protein in vitro. (A) Representative protein levels of p-AMPK, AMPK and NOX4 as determined via western blotting. (B) Semi-quantification of p-AMPK levels. (C) Semi-quantification of NOX4 protein levels. (D) Semi-quantification of AMPK protein levels. (E) Semi-quantification of the p-AMPK/AMPK ratio. (F) Expression of AMPK mRNA as determined via reverse transcription-quantitative PCR. Protein and mRNA levels were normalized to GAPDH. Data are shown as the mean $\pm \mathrm{SEM}(\mathrm{n}=6) .{ }^{\circledR} \mathrm{P}<0.05$, ${ }^{\text {\& }} \mathrm{P}<0.01$ vs. CON + NS-siRNA; ${ }^{*} \mathrm{P}<0.05$ vs. HR + NS-siRNA; ${ }^{\#} \mathrm{P}<0.05,{ }^{\# \prime} \mathrm{P}<0.01$ vs. HR + met + NS-siRNA. CON, control; HR, hypoxia-reoxygenation; met, metformin; siRNA, small interfering RNA; NS-siRNA, non-specific siRNA; AMPK-siRNA, AMPK-specific siRNA; NOX, NADPH oxidase; AMPK, adenosine 5'-monophosphate-activated protein kinase; p, phosphorylated.

The activation of AMPK, which is the downstream factor of metformin (26), was also evaluated. The phosphorylation of AMPK in the IR group was significantly increased compared with that in the Sham group. In the IR + met group, metformin significantly promoted the phosphorylation of AMPK to a higher level than that in the IR group $(\mathrm{P}<0.05$; Fig. $3 \mathrm{~B}$ and $\mathrm{D})$.

AMPK inhibition upregulates the expression of NOX4 protein in vitro. In the NRVM HR model, treatment with metformin increased p-AMPK levels $(\mathrm{P}<0.01$; Fig. $4 \mathrm{~A}$ and $\mathrm{B})$ and significantly reduced NOX4 expression $(\mathrm{P}<0.01$; Fig. $4 \mathrm{~A}$ and $\mathrm{C})$. The AMPK inhibitor compound-C was used to investigate the rela- tionship between AMPK and NOX4; following treatment with compound- $\mathrm{C}$, metformin did not induce a further increase in the phosphorylation of AMPK $(\mathrm{P}<0.05$; Fig. 4B), and the expression of NOX4 was significantly increased compared with the $\mathrm{HR}+$ met group $(\mathrm{P}<0.05$; Fig. 4C).

AMPK-siRNA upregulates the expression of NOX4 protein in vitro. Following transfection with AMPK-siRNA, the expression of AMPK was significantly downregulated in both the CON + AMPK-siRNA group and IR + AMPK-siRNA group compared with the corresponding NS-siRNA groups $(\mathrm{P}<0.05$; Fig. 5F). In the HR + NS-siRNA NRVM 
model, treatment with metformin significantly enhanced AMPK phosphorylation $(\mathrm{P}<0.05$; Fig. 5A and $\mathrm{B})$, while significantly reducing NOX4 expression ( $\mathrm{P}<0.01$; Fig. $5 \mathrm{C})$. AMPK-siRNA was used to explore the relationship between AMPK and NOX4. In the HR + met + AMPK-siRNA group, the expression of AMPK was significantly downregulated compared with the HR + met + NS-siRNA group $(\mathrm{P}<0.01$; Fig. 5A and D). The p-AMPK/AMPK ratio was used to determine the phosphorylation level of AMPK; it was found that p-AMPK/AMPK was significantly increased in the $\mathrm{HR}+$ met + NS-siRNA group compared with in the HR + NS-siRNA group $(\mathrm{P}<0.05$; Fig. 5A and E. No significant difference, however, was observed between the HR + met + NS-siRNA and HR + met + AMPK-siRNA groups $(\mathrm{P}>0.05$; Fig. 5A and E). Following transfection with AMPK-siRNA, metformin did not induce a further increase in $\mathrm{p}$-AMPK $(\mathrm{P}<0.05$; Fig. 5B), and the expression of NOX4 was significantly increased compared with the $\mathrm{HR}+$ met + NS-siRNA group $(\mathrm{P}<0.01$; Fig. 5A and $\mathrm{C})$.

\section{Discussion}

In the present study, the cardioprotective effects of metformin were examined in animal and cell models. Although previous studies had indicated that metformin reduces MIRI $(14,17)$, the specific mechanism remains unclear. In the present study, it was observed that metformin reduced myocardial infarct size, alleviated myocardial oxidative damage and apoptosis, promoted the activation of AMPK and inhibited the expression of NOX4. In primary NRVMs following HR injury, metformin inhibited the expression of NOX4, and this inhibition was reversed using an AMPK inhibitor. These findings indicated that metformin inhibited the expression of NOX4 via the activation of AMPK, thereby reducing oxidative damage and ultimately playing a role in myocardial protection.

It has previously been reported that ischemic postconditioning could effectively reduce myocardial infarct size (29); however, its application is limited. Therefore, there is a motivation to search for medications with potential cardioprotective effects to simulate the effects of postconditioning in a manner that may be more accessible to clinical patients. In the process, it has been found that various endogenous substances, including adenosine, erythropoietin and cell growth factors, play crucial roles in reducing MIRI (30). Another option is to look for existing drugs with cardioprotective effects, expanding their existing uses and greatly reduces the cost of developing new drugs. Statins have been found to exert cardioprotective effects outside of blood lipid regulation, resulting from antioxidant, anti-apoptotic and other effects (31). Previous studies showed that metformin may also have a similar cardioprotective effect. For example, in a prospective diabetes study in the UK, metformin was found to reduce the risk of acute myocardial infarction (AMI) by $39 \%$ in patients with type II diabetes, reducing all-cause mortality by $36 \%$ (32). Regarding patients with AMI, metformin treatment has also significantly reduced their mortality compared with sulfonylureas $(33,34)$. In the present study, it was found that infarct size was significantly reduced in metformin-treated animals in an IR rat model. Consistent with a previous study (16), the results showed that $5 \mathrm{mg} / \mathrm{kg}$ metformin was sufficient for myocardial protection and would not result in changes in blood glucose concentrations.

During MIRI, a large number of intracellular ROS can be generated, which may cause damage to myocardial tissue through various pathways, including destruction of various structural proteins and enzymes, DNA damage, calcium overload and cardiomyocyte apoptosis (35). Previous studies have mainly focused on the effect of metformin on decreasing ROS formation in myocardial fibrosis $(19,36)$. MDA and 4HNE are the products of lipid peroxidation, which reflect the levels of ROS production. In the present study. it was found that metformin could reduce the levels of $4 \mathrm{HNE}$ in a rat model of MIRI, as well as those of MDA in a cell model of HR, thus indicating a protective role.

Intracellular ROS are produced mainly by NOX and mitochondrial pathways (35). It was previously reported that NOX2 and NOX4 are the main sources of cardiac oxygen free radical production and play important roles in the growth and death of cardiomyocytes $(37,38)$. Studies have shown that, following HR treatment, the expression levels of NOX2 are increased $(39,40)$. In addition, cardiac-specific knockout of NOX4 aggravates MIRI (41). In a separate study, there was no significant reduction of IS in rats with NOX4 knockout, suggesting that NOX4 did not aggravate MIRI (7). There remains a degree of controversy concerning the role of NOX4 in MIRI. Therefore, the role of NOX4 in MIRI was investigated in the present study. It was observed that the expression level of NOX4 was increased significantly in the IR group, which is consistent with the finding that $4 \mathrm{HNE}$ levels were significantly increased in the IR group compared with the Sham group. These findings suggested that NOX4 aggravated MIRI by promoting oxidative stress.

A previous study had shown that metformin can inhibit NOX4 activity and alleviate pulmonary fibrosis (36). Similarly, metformin can prevent cardiac fibrosis by inhibiting the activation of NOX, inhibiting the production of ROS (19). These results indicated the potential regulatory association between metformin and NOX4. Metformin also inhibits intracellular oxidation by activating AMPK to stimulate myocardial protection (26). A previous study showed that AMPK alleviated the apoptosis of epithelial cells by inhibiting the activity of NOX4 (20), indicating an association between AMPK and NOX4. As a downstream effector of metformin, increased AMPK phosphorylation has been shown to decrease cardiomyocyte apoptosis and improve cardiac function (15). In the present study, it was found that the phosphorylation of AMPK in the IR group was increased compared with that in the Sham group. After metformin treatment, p-AMPK was significantly increased compared with the IR group, consistent with a previous study (15). In the present study, it was also found that in the metformin group, the mRNA and protein levels of NOX4 were significantly reduced compared with the IR group. These results indicated the association between AMPK and NOX4 in a rat model of MIRI. In the cell model, pretreatment with the AMPK inhibitor compound-C significantly reduced NOX4 protein expression when compared with the group only treated with metformin. To further investigate the role of AMPK in metformin-induced inhibition of NOX4, AMPK expression was knocked down by siRNA transfection. As expected, AMPK-siRNA significantly downregulated the expression of 
AMPK. The p-AMPK/AMPK ratio was used to determine phosphorylation level of AMPK, which was increased by metformin, but was not affected by transfection with NS-siRNA or AMPK-siRNA in metformin-treated HR cells. Evaluating p-AMPK in isolation, it was observed that AMPK-siRNA reduced p-AMPK levels compared with NS-siRNA, whilst also increasing the expression of NOX4 (Fig. 5C). These findings suggested that the regulation of NOX4 activity depends on the levels of activated AMPK. In conclusion, the results of the present study indicated that metformin inhibited the expression of NOX4 via the activation of AMPK, leading to a reduction of myocardial oxidative damage, apoptosis and infarct size, ultimately alleviating MIRI.

\section{Acknowledgements}

Not applicable.

\section{Funding}

No funding was received.

\section{Availability of data and materials}

All data generated or analyzed during this study are included in this published article.

\section{Authors' contributions}

YS and SAH conceived and designed the study. YS conducted both the in vivo and in vitro studies and drafted the manuscript. SAH assisted in drafting the manuscript. YS and SAH confirm the authenticity of all the raw data. All authors read and approved the final manuscript.

\section{Ethics approval and consent to participate}

The Guide for the Care and Use of Laboratory Animals was followed, and the experimental protocol was approved by the Local Ethics Committee at the Medical College of Shandong University.

\section{Patient consent for publication}

Not applicable.

\section{Competing interests}

The authors declare that they have no competing interests.

\section{References}

1. Malakar AK, Choudhury D, Halder B, Paul P, Uddin A and Chakraborty S: A review on coronary artery disease, its risk factors, and therapeutics. J Cell Physiol 234: 16812-16823, 2019.

2. Monassier JP: Reperfusion injury in acute myocardial infarction. From bench to cath lab. Part I: Basic considerations. Arch Cardiovasc Dis 101: 491-500, 2008.

3. Yellon DM and Hausenloy DJ: Myocardial reperfusion injury. N Engl J Med 357: 1121-1135, 2007.

4. Sies H: Oxidative stress: A concept in redox biology and medicine. Redox Biol 4: 180-183, 2015.
5. Matsushima S, Tsutsui H and Sadoshima J: Physiological and pathological functions of NADPH oxidases during myocardial ischemia-reperfusion. Trends Cardiovasc Med 24: 202-205, 2014.

6. Braunersreuther V, Montecucco F, Asrih M, Pelli G, Galan K, Frias M, Burger F, Quinderé AL, Montessuit C, Krause KH, et al: Role of NADPH oxidase isoforms NOX1, NOX2 and NOX4 in myocardial ischemia/reperfusion injury. J Mol Cell Cardiol 64: 99-107, 2013.

7. Matsushima S, Kuroda J, Ago T, Zhai P, Ikeda Y, Oka S, Fong GH, Tian R and Sadoshima J: Broad suppression of NADPH oxidase activity exacerbates ischemia/reperfusion injury through inadvertent downregulation of HIF-1 $\alpha$ and upregulation of PPAR $\alpha$. Circ Res 112: 315-325, 2013.

8. Russell RR III, Li J, Coven DL, Pypaert M, Zechner C, Palmeri M, Giordano FJ, Mu J, Birnbaum MJ and Young LH: AMP-activated protein kinase mediates ischemic glucose uptake and prevents postischemic cardiac dysfunction, apoptosis, and injury. J Clin Invest 114: 495-503, 2004

9. Folmes CD, Wagg CS, Shen M, Clanachan AS, Tian R and Lopaschuk GD: Suppression of 5'-AMP-activated protein kinase activity does not impair recovery of contractile function during reperfusion of ischemic hearts. Am J Physiol Heart Circ Physiol 297: H313-H321, 2009.

10. Liu B, Clanachan AS, Schulz R and Lopaschuk GD: Cardiac efficiency is improved after ischemia by altering both the source and fate of protons. Circ Res 79: 940-948, 1996.

11. Wang YW, He SJ, Feng X, Cheng J, Luo YT, Tian L and Huang Q: Metformin: A review of its potential indications. Drug Des Devel Ther 11: 2421-2429, 2017.

12. Nicholls SJ, Tuzcu EM, Kalidindi S, Wolski K, Moon KW, Sipahi I, Schoenhagen P and Nissen SE: Effect of diabetes on progression of coronary atherosclerosis and arterial remodeling: A pooled analysis of 5 intravascular ultrasound trials. J Am Coll Cardiol 52: 255-262, 2008.

13. Norhammar A,Lindbäck J, Rydén L, Wallentin L and Stenestrand U; Register of Information and Knowledge about Swedish Heart Intensive Care Admission (RIKS-HIA): Improved but still high short- and long-term mortality rates after myocardial infarction in patients with diabetes mellitus: A time-trend report from the Swedish Register of Information and Knowledge about Swedish Heart Intensive Care Admission. Heart 93: 1577-1583, 2007.

14. Wang X, Yang L, Kang L, Li J, Yang L, Zhang J, Liu J, Zhu M, Zhang Q, Shen Y, et al: Metformin attenuates myocardial ischemia-reperfusion injury via up-regulation of antioxidant enzymes. PLoS One 12: e0182777, 2017.

15. Calvert JW, Gundewar S, Jha S, Greer JJ, Bestermann WH, Tian R and Lefer DJ: Acute metformin therapy confers cardioprotection against myocardial infarction via AMPK-eNOS-mediated signaling. Diabetes 57: 696-705, 2008.

16. Paiva M, Riksen NP, Davidson SM, Hausenloy DJ, Monteiro P, Gonçalves L, Providência L, Rongen GA, Smits P, Mocanu MM, et al: Metformin prevents myocardial reperfusion injury by activating the adenosine receptor. J Cardiovasc Pharmacol 53: 373-378, 2009.

17. Solskov L, L $\varnothing$ fgren B, Kristiansen SB, Jessen N, Pold R, Nielsen TT, Bøtker HE, Schmitz O and Lund S: Metformin induces cardioprotection against ischaemia/reperfusion injury in the rat heart 24 hours after administration. Basic Clin Pharmacol Toxicol 103: 82-87, 2008.

18. Melendez GC, Chen Q and Lesnefsky EJ: Metformin as a modulator of myocardial fibrosis post-myocardial infarction via regulation of cardiomyocyte-fibroblast crosstalk. Transl Res 199: 1-3, 2018.

19. Bai J, Zhang N, Hua Y, Wang B, Ling L, Ferro A and Xu B: Metformin inhibits angiotensin II-induced differentiation of cardiac fibroblasts into myofibroblasts. PLoS One 8: e72120,2013.

20. Eid AA, Ford BM, Block K, Kasinath BS, Gorin Y, Ghosh-Choudhury G, Barnes JL and Abboud HE: AMP-activated protein kinase (AMPK) negatively regulates Nox4-dependent activation of p53 and epithelial cell apoptosis in diabetes. J Biol Chem 285: 37503-37512, 2010.

21. Zhang WX, Tai GJ, Li XX and Xu M: Inhibition of neointima hyperplasia by the combined therapy of linagliptin and metformin via AMPK/Nox4 signaling in diabetic rats. Free Radic Biol Med 143: 153-163, 2019.

22. National Research Council: Guide for the Care and Use of Laboratory Animals. National Academy Press, Washington, DC, 1985.

23. Lee SY, Ku HC, Kuo YH, Chiu HL and Su MJ: Pyrrolidinyl caffeamide against ischemia/reperfusion injury in cardiomyocytes through AMPK/AKT pathways. J Biomed Sci 22: 18, 2015. 
24. Cheng Y, Zhu P, Yang J, Liu X, Dong S, Wang X, Chun B, Zhuang $J$ and Zhang $C$ : Ischaemic preconditioning-regulated miR-21 protects heart against ischaemia/reperfusion injury via anti-apoptosis through its target PDCD4. Cardiovasc Res 87: 431-439, 2010.

25. Sun HY, Wang NP, Kerendi F, Halkos M, Kin H, Guyton RA, Vinten-Johansen J and Zhao ZQ: Hypoxic postconditioning reduces cardiomyocyte loss by inhibiting ROS generation and intracellular $\mathrm{Ca}^{2+}$ overload. Am J Physiol Heart Circ Physiol 288: H1900-H1908, 2005.

26. Kobashigawa LC, Xu YC, Padbury JF, Tseng YT and Yano N Metformin protects cardiomyocyte from doxorubicin induced cytotoxicity through an AMP-activated protein kinase dependent signaling pathway: An in vitro study. PLoS One 9: e104888, 2014.

27. Livak KJ and Schmittgen TD: Analysis of relative gene expression data using real-time quantitative PCR and the 2(-Delta Delta C(T)) Method. Methods 25: 402-408, 2001

28. Poli G and Schaur JR: 4'Hydroxynonenal in the pathomechanisms of oxidative stress. IUBMB Life 50: 315-321, 2000.

29. Ibáñez B, Heusch G, Ovize M and Van de Werf F: Evolving therapies for myocardial ischemia/reperfusion injury. J Am Coll Cardiol 65: 1454-1471, 2015.

30. Sluijter JP, Condorelli G, Davidson SM, Engel FB, Ferdinandy P, Hausenloy DJ, Lecour S, Madonna R, Ovize M, Ruiz-Meana M, et al; Nucleus of the European Society of Cardiology Working Group Cellular Biology of the Heart: Novel therapeutic strategies for cardioprotection. Pharmacol Ther 144: 60-70, 2014.

31. Hadi NR, Al-Amran F, Yousif M and Zamil ST: Antiapoptotic effect of simvastatin ameliorates myocardial ischemia/reperfusion injury. ISRN Pharmacol 2013: 815094, 2013.

32. Group UPDS; UK Prospective Diabetes Study (UKPDS) Group Effect of intensive blood-glucose control with metformin on complications in overweight patients with type 2 diabetes (UKPDS 34). Lancet 352: 854-865, 1998.

33. Schramm TK, Gislason GH, Vaag A, Rasmussen JN, Folke F, Hansen ML, Fosb $\varnothing 1$ EL, Køber L, Norgaard ML, Madsen M, et al: Mortality and cardiovascular risk associated with different insulin secretagogues compared with metformin in type 2 diabetes, with or without a previous myocardial infarction: A nationwide study. Eur Heart J 32: 1900-1908, 2011.
34. Masoudi FA, Inzucchi SE, Wang Y, Havranek EP, Foody JM and Krumholz HM: Thiazolidinediones, metformin, and outcomes in older patients with diabetes and heart failure: An observational study. Circulation 111: 583-590, 2005.

35. Bedard K and Krause KH: The NOX family of ROS-generating NADPH oxidases: physiology and pathophysiology. Physiol Rev 87: 245-313, 2007.

36. Sato N, Takasaka N, Yoshida M, Tsubouchi K, Minagawa S, Araya J, Saito N, Fujita Y, Kurita Y, Kobayashi K, et al: Metformin attenuates lung fibrosis development via NOX4 suppression. Respir Res 17: 107, 2016.

37. Murdoch CE, Grieve DJ, Cave AC, Looi YH and Shah AM: NADPH oxidase and heart failure. Curr Opin Pharmacol 6: 148-153, 2006.

38. Sirker A, Zhang M and Shah AM: NADPH oxidases in cardiovascular disease: Insights from in vivo models and clinical studies. Basic Res Cardiol 106: 735-747, 2011.

39. Cheng F, Yuan W, Cao M, Chen R, Wu X, Yan J and Cyclophilin A: Cyclophilin a protects cardiomyocytes against hypoxia/reoxygenation-induced apoptosis via the AKT/Nox2 pathway. Oxid Med Cell Longev 2019: 2717986, 2019.

40. Jin Q, Jiang Y, Fu L, Zheng Y, Ding Y and Liu Q: Wenxin granule ameliorates hypoxia/reoxygenation-induced oxidative stress in mitochondria via the $\mathrm{PKC}-\delta / \mathrm{NOX} 2 / \mathrm{ROS}$ pathway in $\mathrm{H} 9 \mathrm{c} 2$ cells Oxid Med Cell Longev 2020: 3245483, 2020.

41. Martyn KD, Frederick LM, von Loehneysen K, Dinauer MC and Knaus UG: Functional analysis of Nox4 reveals unique characteristics compared to other NADPH oxidases. Cell Signal 18: 69-82, 2006

This work is licensed under a Creative Commons Attribution-NonCommercial-NoDerivatives 4.0 International (CC BY-NC-ND 4.0) License. 